\title{
A real-time, nonintrusive trace gas detector based on laser photothermal deflection
}

\author{
H. S. M. de Vries, N. Dam, M. R. van Lieshout, C. Sikkens, F. J. M. Harren, and J. \\ Reuss \\ Department of Molecular and Laser Physics, Catholic University of Nijmegen, Toernooiveld, NL-6525 ED \\ Nijmegen, The Netherlands
}

(Received 20 March 1995; accepted for publication 16 May 1995)

\begin{abstract}
We present the layout and technical details of a trace gas monitor based on photothermal deflection. The operating principle of this instrument, i.e., the deflection of a (weak) probe laser beam by the thermal refractive index gradient induced by trace gas absorption of an intense pump laser beam, allows nonintrusive measurements with good space and time resolution. An intra-cavity $\mathrm{CO}_{2}$ laser is used as the pump beam and a red HeNe laser as the probe. The latter runs perpendicular to the pump beam to optimize spatial resolution. To increase sensitivity, the probe laser is incorporated in a multipass setup. The instrument is demonstrated by the localization of ethylene emission sites on a cherry tomato and by monitoring ammonia production due to nitrogen fixation by cyanobacteria. Both $\mathrm{C}_{2} \mathrm{H}_{4}$ and $\mathrm{NH}_{3}$ can be detected at the $1-3 \mathrm{ppb}$ level, at a spatial resolution of $2 \mathrm{~mm}$ (along the pump laser) $\times 0.6 \mathrm{~mm}$ (perpendicular to it), and a response time of $0.1 \mathrm{~s}$ (without background correction) or $15 \mathrm{~s}$ (including background correction). Sensitivity can be increased at the expense of spatial resolution, and vice versa. In principle, this instrument is applicable to all those gases possessing a characteristic ("fingerprint") spectrum in the $\mathrm{CO}_{2}$ laser range. The great advantage of the photothermal deflection technique with respect to other trace gas detection schemes lies in the nonintrusive character of the measurements. There is no need to enclose the sample in a vessel or to suck large volumes of air into the detector; measurements can be performed in open air and in real time. This should prove especially useful where sticky (polar) gases, like $\mathrm{H}_{2} \mathrm{O}, \mathrm{NH}_{3}, \mathrm{CH}_{3} \mathrm{OH}$, etc., are to be detected quantitatively. Main applications include air quality monitoring, especially concerning dry deposition rate measurements using the eddy correlation technique, and the study of volatile metabolite emission of biological samples. (C) 1995 American Institute of Physics.
\end{abstract}

\section{INTRODUCTION}

Many applications in environmental, biological and medical science require instrumentation capable of detecting low concentrations of specific volatile compounds (trace gases) in air with high spatial and temporal resolution. Many solutions have been offered as well. Among these, the gas chromatograph $^{1,2}$ and the laser photoacoustic method ${ }^{3-6}$ may well be the most successful. Both methods, however, still compromise time and space resolution because active sampling is required to transfer the sample to the detection unit. This may pose a problem, especially when dipolar (=sticky) gases are involved, like ammonia $\left(\mathrm{NH}_{3}\right)$ or water $\left(\mathrm{H}_{2} \mathrm{O}\right)$.

These problems can be circumvented by the nonintrusive photodeflection method, which is based on the deflection of a (laser) light beam by a local refractive index gradient created by the absorption of another (laser) light beam by the trace gas of interest. The photothermal deflection (PTD) technique has been applied in many fields of research, ${ }^{7}$ e.g., semiconductor physics, ${ }^{8-10}$ liquid ${ }^{11,12}$ and solid state physics, ${ }^{13-16}$ medical physics, ${ }^{17,18}$ flame spectroscopy, ${ }^{19}$ and in exhaust gas analysis. ${ }^{20}$ Its potential for trace gas detection was mentioned only briefly for ethylene. ${ }^{21,22}$ The general theory of PTD has been described by Murphy ${ }^{23}$ and Jackson. ${ }^{24}$ Charbonnier ${ }^{25}$ published the first design for a practical deflection setup.

We have developed a $\mathrm{CO}_{2}$ laser-driven PTD instrument and applied it to the in situ detection of ethylenc and ammo- nia at ppbv levels ( $1 \mathrm{ppbv}=1: 10^{9}$ molecules). The layout and technical details of the setup and its first results will be described below.

To illustrate the possibilities offered by our newly developed instrument, we have chosen two applications to biological and environmental sciences. The first of these concerns the local emission of ethylene $\left(\mathrm{C}_{2} \mathrm{H}_{4}\right)$ by living plants. Ethylene is a plant hormone involved in many regulatory functions like germination, growth and senescence of plants, ripening of fruit and wilting of flowers, ${ }^{26}$ and as such of paramount interest to plant physiologists and commercial fruit and flower growers alike. We have chosen the tomato as a test object because a considerable amount of earlier data on its $\mathrm{C}_{2} \mathrm{H}_{4}$ emission are available for comparison. ${ }^{27-30}$

Our second example, highlighting the time resolution and nonintrusive character of our detector, concerns the detection of ammonia, $\mathrm{NH}_{3}$. Ammonia owns most of its (bad) reputation from its role in soil acidification. For certain ecosystems, ambient $\mathrm{NH}_{3}$ concentrations of only $100 \mathrm{ppbv}$ can already be disastrous. ${ }^{31}$ The measurement of dry deposition rates by means of the eddy correlation technique ${ }^{32}$ requires local $\mathrm{NH}_{3}$ concentration measurements at $10 \mathrm{~Hz}$ repetition rate. We demonstrate the response time of our system by exposing it to artificial pulses of ammonia in air, showing that PTD should be suitable for eddy correlation measurements. 

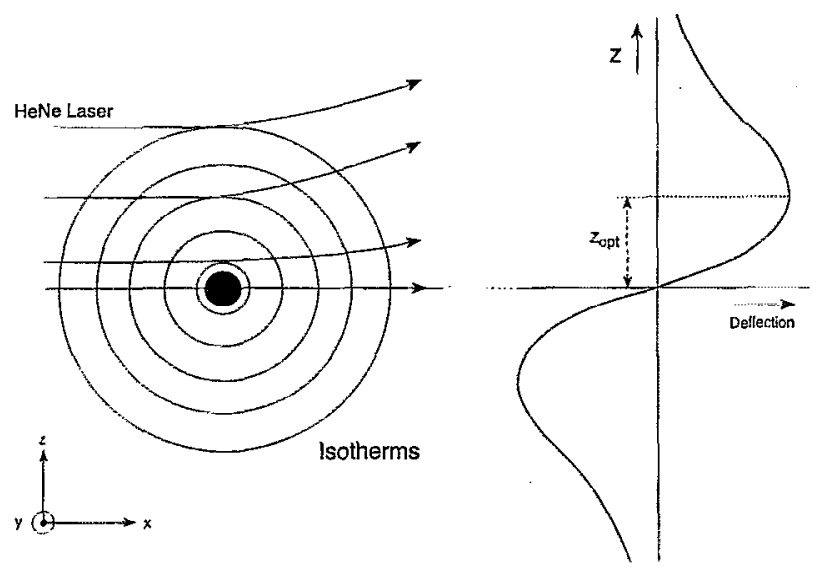

FIG. 1. The principle of (transverse) photothermal deflection. Absorption of a Gaussian pump laser beam (large central dot) induces a temperature gradient with concentrical isotherms. The associated refractive index gradient is employed to refract a second ( $\mathrm{HeNe}$ ) laser beam; the deflection angle is a direct measure of the amount of pump laser light absorbed. $Z_{\text {opt }}$ corresponds to the distance between pump and probe beam at which the maximum refractive index gradient occurs.

\section{THEORY OF PTD}

Detection of light absorption can proceed in two ways, either by detecting changes in the transmitted light beam or by detecting changes induced in the absorbing medium by the light beam. The well-known technique of linear absorption spectroscopy belongs to the former class. Although conceptually straightforward and easily interpreted, it often suffers from having to detect small absorption signals against the large background of the unattenuated light beam. All ultrasensitive detection techniques, therefore, seek to detect changes induced in the absorbing medium as a result of light absorption. Examples are laser induced fluorescence (LIF), Photoacoustics (PA), and PTD, to be discussed here.

The principle of the PTD technique (Fig. 1) relies on the deflection of a light beam when it traverses a (gaseous) medium with a refractive index gradient perpendicular to the propagation direction of the beam. In PTD, this refractive index gradient is induced by an intense light beam, which will be called the pump beam. Absorption of the pump beam by the medium gives rise to a local temperature increase, leading to a temperature gradient with concentric, cylindrical isotherms. According to the ideal gas law, this temperature gradient is accompanied by a density gradient and, therefore, by a refractive index gradient. The latter is probed by measuring the deflection of a second light beam, the probe; the deflection is a direct measure of the amount of pump light absorbed. Thus, the probe deflection is proportional to the intensity of the pump beam and to the density of the absorbing species in the medium (which may be heterogeneous). Both pump and probe beam are most conveniently derived from laser sources, providing a narrow-bandwidth, highintensity pump beam and a highly collimated probe beam with good pointing stability.

In the following we will calculate the deflection angle of the probe beam (a HeNe laser) due to a refractive index gradient induced by a strong pump laser $\left(\mathrm{CO}_{2}\right.$ laser $)$ in a gaseous medium containing trace amounts of absorbing gas.
Rather than attempting to derive a general formalism (for this, see Jackson ${ }^{24}$ ), we restrict ourselves to the experimental conditions yielding the best spatial resolution (that is, transverse PTD $^{24}$ ) and will try to identify those experimentally accessible parameters that are most decisive for instrument performance. Also, the effects of amplitude modulation of the pump laser, which allows noise suppression through lock-in detection techniques, are considered in some detail.

The solution of the diffusion equation for an instantaneous point source, given by Crank, ${ }^{33}$ can be generalized to the position and time-dependent energy density, $W(\mathbf{r}, t)$, in the presence of an extended, continuous source,

$$
\begin{aligned}
W(\vec{r}, t)= & \int_{-\infty}^{t} \int_{\text {space }} \frac{\Delta E\left(\vec{r}^{\prime}, t^{\prime}\right)}{8\left[\pi \kappa\left(t-t^{\prime}\right)\right]^{3 / 2}} \\
& \times \exp \left[\frac{-\left|\vec{r}-\vec{r}^{\prime}\right|^{2}}{4 \kappa\left(t-t^{\prime}\right)}\right] d^{3} r^{\prime} d t^{\prime}+W_{0} .
\end{aligned}
$$

Here, $W_{0}$ denotes the unperturbed energy density, $\kappa$ is the heat diffusion coefficient (in $\left.\mathrm{m}^{2} \mathrm{~s}^{-1}\right)$, and $\Delta E\left(\mathbf{r}^{\prime}, t^{\prime}\right)$ is to be interpreted as the energy density rise per unit time due to local energy input. This energy input is equal to the energy lost from the pump beam by absorption, so that we can write

$$
\begin{aligned}
\Delta E\left(\vec{r}^{\prime}, t^{\prime}\right) & =-\frac{d W_{p}\left(\vec{r}^{\prime}, t^{\prime}\right)}{d t^{\prime}} \\
& =-\frac{d I_{p}\left(\vec{r}^{\prime}, t^{\prime}\right)}{d y^{\prime}} \\
& =\alpha\left(I_{p}\right) p_{a}\left(\vec{r}^{\prime}, t^{\prime}\right) I_{p}\left(\vec{r}^{\prime}, t^{\prime}\right) .
\end{aligned}
$$

Here, $W_{p}$ denotes the pump laser energy density, $I_{p}$ its intensity, $\alpha$ the (pump laser intensity dependent) absorption coefficient $\left(\mathrm{Pa}^{-1} \mathrm{~m}^{-1}\right){ }^{34}$ and $p_{a}$ the partial pressure $(\mathrm{Pa})$ of the absorbing gas. The pump laser is taken to propagate along the $\hat{y}$ axis (see Fig. 1 for the coordinate system). In air, the local energy density is related to the local temperature $T$ by

$$
W(\vec{r}, t)=\frac{5}{2} k_{b} T(\vec{r}, t) \rho_{T},
$$

with $\rho_{T}$ the total density (particles $/ \mathrm{m}^{3}$ ) and $k_{b}$ is Boltzmann's constant. The contribution of the vibrational degrees of freedom is negligible (at room temperature). Using the ideal gas law to replace $p_{a}$ in Eq. (2) by $k_{b} T \rho_{a}$, with $\rho_{a}$ the absorbing gas particle density, we find

$$
\begin{aligned}
T(\vec{r}, t)= & \int_{-\infty}^{t} \int_{\text {all }} \frac{\alpha(I) \rho_{a}\left(\vec{r}^{\prime}, t^{\prime}\right) I_{p}\left(\vec{r}^{\prime}, t^{\prime}\right)}{20 \rho_{T}\left[\pi \kappa\left(t-t^{\prime}\right)\right]^{3 / 2}} \\
& \times T\left(\vec{r}^{\prime}, t^{\prime}\right) \exp \left[\frac{-\left|\vec{r}-\vec{r}^{\prime}\right|^{2}}{4 \kappa\left(t-t^{\prime}\right)}\right] d^{3} r^{\prime} d t^{\prime}+T_{\text {amb }},
\end{aligned}
$$

with $T_{\text {amb }}$ the ambient temperature. For the small absorptions that we will consider, the temperature rise above ambient is so small (a few mK), that $T\left(\vec{r}^{\prime}, t^{\prime}\right)$ can be replaced by $T_{\text {amb }}$ in the integrand. Assuming the laser to operate in a pure $\mathrm{TEM}_{00}$ mode, its position dependent intensity profile can be written as $^{35}$

$$
I_{p}\left(\vec{r}^{\prime}, t^{\prime}\right)=\frac{2 P_{w}\left(t^{\prime}\right)}{\pi w^{2}\left(y^{\prime}\right)} \exp \left[-\frac{2\left(x^{\prime 2}+z^{\prime 2}\right)}{w^{2}\left(y^{\prime}\right)}\right],
$$


where $P_{w}$ denotes the laser power (Watt) and $w\left(y^{\prime}\right)$ the laser beam waist (1/e radius of the electric field strength). In the actual experiments, the laser beam is focused to a spot size of $w_{0}=0.28 \mathrm{~mm}$. The corresponding Rayleigh length amounts to $25 \mathrm{~mm}$ (for $\lambda=10 \mu \mathrm{m}$ ), which is much larger than all other relevant distances in the problem. We therefore take $w\left(y^{\prime}\right)=w_{0}$ in the whole region of interest. ${ }^{36}$

Two other approximations will be made. First of all, we will neglect the exact intensity dependence of the absorption coefficient. Rather, for those instances where saturation is important, the absorption coefficient will be replaced by an effective one, which can be determined experimentally. Furthermore, the density $\rho_{a}$ will be assumed constant over that part of the pump laser beam which contributes most significantly to the refractive index gradient at the site of the probe beam. With these assumptions, the space integral of Eq. (4) can be performed analytically, yielding

$$
\begin{aligned}
T(\vec{r}, t)= & \frac{4 \alpha \rho_{a}}{5 \pi \rho_{T}} T_{\text {amb }} \int_{-\infty}^{t} \frac{P_{w}\left(t^{\prime}\right)}{8 \kappa\left(t-t^{\prime}\right)+w_{0}^{2}} \\
& \times \exp \left[-\frac{2\left(x^{2}+z^{2}\right)}{8 \kappa\left(t-t^{\prime}\right)+w_{0}^{2}}\right] d t^{\prime}+T_{\text {amb }} .
\end{aligned}
$$

In the experiments, the pump laser is mechanically chopped (frequency $\nu_{\text {chop }}$ ), that is, $P_{w}\left(t^{\prime}\right)$ is a block function with period $\tau=1 / \nu_{\text {chop }}$. Taking $t=0$ at the rising edge of the last chopper period, the time integral of Eq. (6) can be evaluated. This yields the following expression for the induced temperature rise, $\Delta T=T(\vec{r}, t)-T_{\mathrm{amb}}$ :

$$
\begin{aligned}
\Delta T(\vec{r}, t)= & \frac{\alpha \rho_{a} P_{0}}{10 \pi \kappa \rho_{T}} T_{\mathrm{amb}} \sum_{n=0}^{\infty}\left\{\mathscr{E}_{1}[u(\vec{r}, t,-n \tau)]\right. \\
& \left.-\mathscr{E}_{1}[u(\vec{r}, t, \min [t,-(n-1 / 2) \tau])]\right\},
\end{aligned}
$$

with $P_{0}$ the nominal laser power, the function $\min \left(t_{1}, t_{2}\right)$ returns the smallest of its two arguments, $\mathscr{E}_{1}(u)=\int_{u}^{\infty} x^{-1} e^{-x} d x$ is an exponential integral ${ }^{37}$ and we have defined

$$
u\left(\vec{r}, t_{1}, t_{2}\right)=\frac{x^{2}+z^{2}}{8 \kappa\left(t_{1}-t_{2}\right)+w_{0}^{2}} .
$$

The $n=0$ term in Eq. (7) gives the contribution of the last chopper cycle to the induced temperature rise; the contributions of all previous cycles are contained in the other terms of the summation. For low chopper frequencies, the temperature reaches an equilibrium during each laser on/off halfperiod, so that effectively only the last chopping. cycle contributes to $\Delta T$. Equation (7) then reduces to

$$
\begin{aligned}
\Delta T(\vec{r}, t)= & \frac{\alpha \rho_{a} P_{0}}{10 \pi \kappa \rho_{T}} T_{\mathrm{amb}} \\
& \times \begin{cases}\mathscr{E}_{1}[u(\vec{r}, t, 0)]-\mathscr{E}_{1}[u(\vec{r}, t, t)], & 0<t<\tau / 2 \\
\mathscr{E}_{1}[u(\vec{r}, t, 0)]-\mathscr{E}_{1}[u(\vec{r}, t, \tau / 2)], & \tau / 2<t<\tau\end{cases}
\end{aligned}
$$

The typical course of the temperature profile [Eq. (7)] as a function of distance from the pump laser beam during one chopper cycle is illustrated in Fig. 2. Evidently, the tempera-

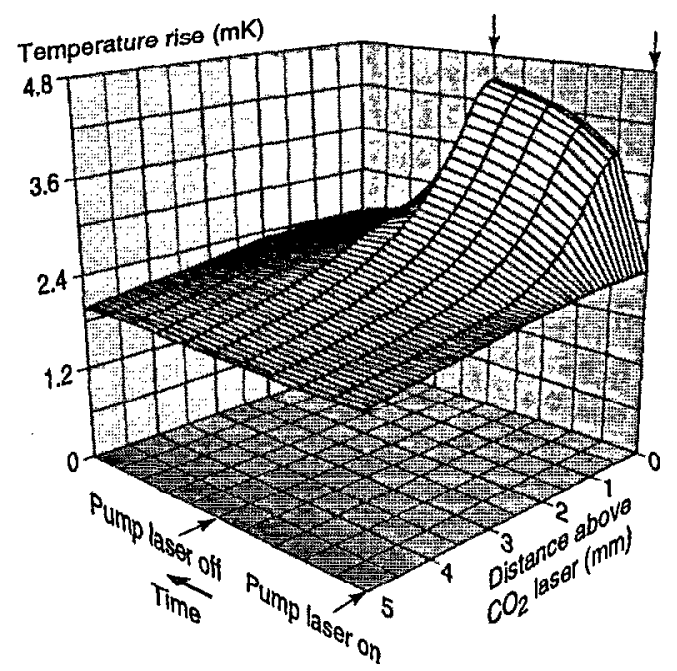

FIG. 2. Calculated temperature rise as a function of distance from the pump laser beam during one chopper period $(50 \mathrm{~ms})$. Parameters used are 1 ppbv of ethylene in air at room temperature, $\kappa=2.05 \times 10^{-5} \mathrm{~m}^{2} \mathrm{~s}^{-1}$ (see Ref. 53) and $100 \mathrm{~W}$ intracavity $\mathrm{CO}_{2}$ laser power (10P14, at which $\alpha=0.0131$ $\mathrm{Pa}^{-1} \mathrm{~m}^{-1}$, see Table III).

ture profile has the same symmetry as the laser beam, that is, the isotherms are concentrical cylinders around the pump axis.

The temperature rise modifies the local refractive index in two ways. On the one hand, it causes a slight redistribution of population over the molecular energy levels and, thus, a slight change in the susceptibility of the medium as a whole. On the other hand, through the ideal gas law, the temperature rise will be accompanied by a density decrease. This density change is directly proportional to a refractive index change, so that the latter effect is expected to far outweigh the former. We then have

$$
\Delta n(\vec{r}, t)=-a \Delta T(\vec{r}, t),
$$

with the proportionality constant $a=2.3 \times 10^{-7} \mathrm{~K}^{-1}$ for dry air (STP@ $\lambda=10 \mu \mathrm{m}$; extrapolated from ${ }^{30}$ ).

The probe laser deflection will be calculated for the case that it passes at a nominal height $z_{h}$ above the pump laser and propagates along the $\hat{x}$ axis, that is, perpendicular to the pump laser (Fig. 1). This is the configuration yielding the best spatial resolution. We will use geometrical optics to calculate the deflection. This is a considerable simplification as compared to the case of thermal lensing, where the focusing properties of the heated medium must be calculated and Gaussian beam optics must be used.

Consider first the refraction of the probe beam at one specific "isofract" (contour of constant refractive index) at a distance $r_{h}=\sqrt{x_{h}^{2}+z_{h}^{2}}$ from the pump beam (Fig. 3). From Snell's law we can relate the angle of refraction $u$ to the angle of incidence $i$ by

$$
n\left(r_{h}+d x_{h}\right) \sin i=n\left(r_{h}\right) \sin u .
$$

Writing $u=i+d i$, with $d i \ll 1$ and expanding $n\left(r_{h}+d x_{h}\right)$ to first order yields

$$
d i=\tan i\left(x_{h}\right) \frac{\partial n\left(r_{h}\right)}{\partial x_{h}} d x_{h} .
$$




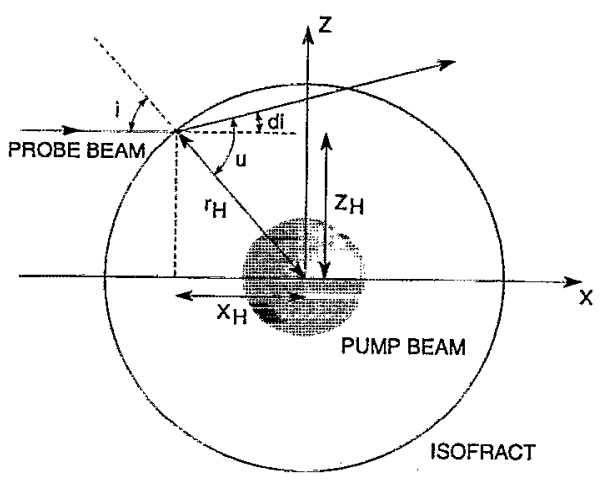

FIG. 3. Deflection of the probe laser on one specific contour of constant refractive index ("isofract"). The pump laser propagates perpendicular to the plane of the figure. The symbols are explained in the text.

For the applications discussed in this article, the total deflection angle is always very small, so that the probe beam can be assumed always to be parallel to the $\hat{x}$ axis. Then, using Eqs. (7) and (10), the total deflection angle $\Delta i$, can be expressed in terms of error functions,

$$
\begin{aligned}
\Delta i(t)= & \frac{a \alpha \rho_{a} P_{0}}{5 \kappa \rho_{T}} T_{\mathrm{amb}} \sum_{n=0}^{\infty}\left[\operatorname{erf}\left(g\left\{z_{h}, \max \left[0, t+\left(n-\frac{1}{2}\right) \tau\right]\right\}\right)\right. \\
& \left.-\operatorname{erf}\left(g\left\{z_{h}, t+n \tau\right\}\right)\right],
\end{aligned}
$$

with

$$
g\left(z_{h}, \Delta t\right)=\sqrt{\frac{2 z_{h}^{2}}{8 \kappa \Delta t+w_{0}^{2}}} .
$$

The deflection of the probe beam is lock-in detected, and the measured signal $S$ is therefore

$$
\begin{aligned}
S= & \frac{2 \eta}{\tau} \int_{0}^{\tau / 2} \Delta i(t) d t-\frac{2 \eta}{\tau} \int_{\tau / 2}^{\tau} \Delta i(t) d t \\
= & \frac{a \alpha \rho_{a} P_{0}}{3 \kappa \rho_{T}} \eta T_{\text {amb }}\left\{\operatorname{erf} \sqrt{\frac{2 z_{h}^{2}}{w_{0}^{2}}}+2 \wp[0]+\wp(\tau)\right. \\
& -3 \wp(\tau / 2)+\sum_{n=1}^{\infty}[3 \wp[n \tau]+\wp[(n+1) \tau] \\
& \left.-3 \wp\left[\left(n+\frac{1}{2}\right) \tau\right]-\wp\left[\left(n-\frac{1}{2}\right) \tau\right]\right\},
\end{aligned}
$$

with

$$
\begin{aligned}
\wp[\Delta t]= & \frac{z_{h}^{2}}{\tau \kappa}\left\{\frac{\exp \left[-g^{2}\left(z_{h}, \Delta t\right)\right]}{\sqrt{\pi} g\left(z_{h}, \Delta t\right)}\right. \\
& \left.+\left(1+\frac{1}{2 g^{2}\left(z_{h}, \Delta t\right)}\right) \operatorname{erf}\left[g\left(z_{h}, \Delta t\right)\right]\right\} .
\end{aligned}
$$

Here, $\eta$ denotes the detector sensitivity. Equation (15) can be used to calculate the photothermal deflection signal under given experimental conditions. An example is given in Fig. 4 (lower part), for different chopper frequencies. The qualitative agreement with the experimental curves (Fig. 4, upper part) is seen to be quite satisfactory, but quantitatively there are some discrepancies. Experimentally, the maximum signal amplitude is found at larger distances between the two laser beams and the signal decreases much faster with increasing
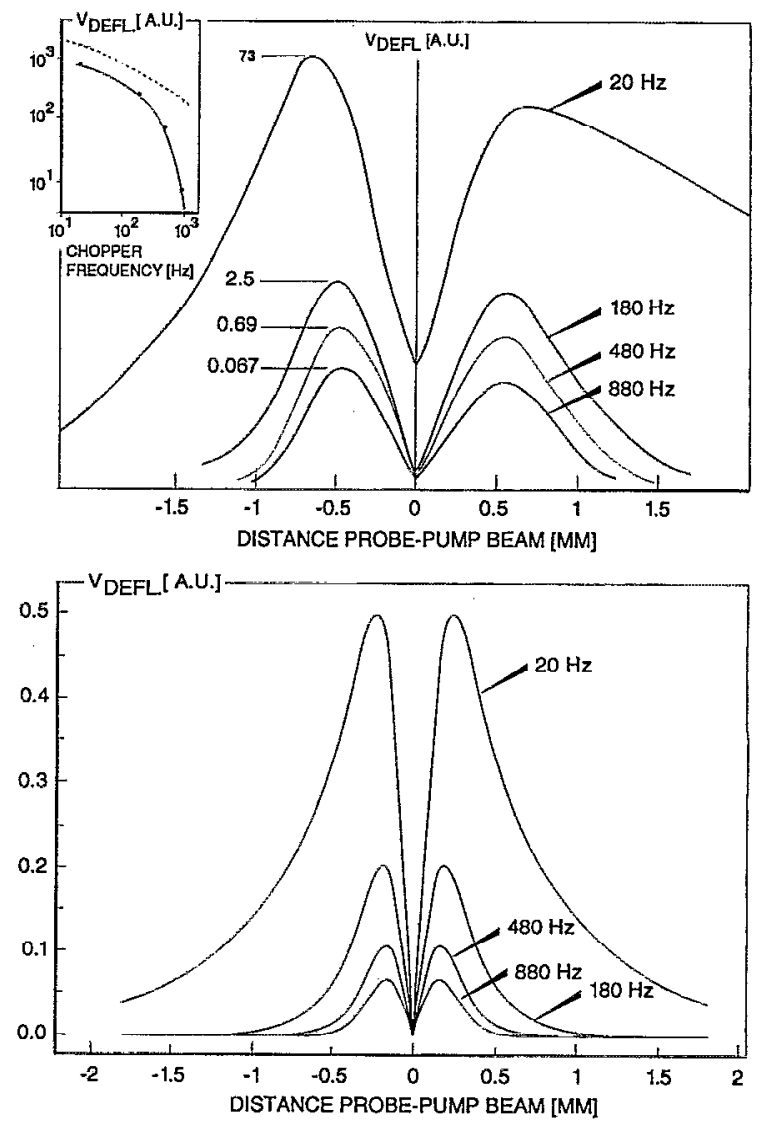

FIG. 4. Calculated and experimental lock-in detected deflection signals. Upper part: Experimental curves for several chopper frequencies. Note that the ordinate is not to scale; the numbers at the left of the curves indicate peak values. The insert shows the maximum signal strength as a function of chopper frequency for experimental (solid line) and theoretical (dashed line) data. Lower part: Calculated from Eq. (15) with the experimental parameters of Fig. 2 and the same chopper frequencies as used in the experiment.

chopper frequency. Both deviations can be attributed to the relatively large dimensions of the probe laser beam (waist of $0.4 \mathrm{~mm}$ ), which effectively implies that a weighted average of deflections over a range of $z_{h}$ is actually measured. Since the deflection angle changes sign on crossing the $x y$ plane, this averaging results in an outward shift of the position where maximum signal will be found. Moreover, since the extent of the temperature gradient (Fig. 2) quickly diminishes with increasing chopper frequency, the signal decrease will be faster for a (practical) Gaussian probe beam than for an (ideal) line-shaped probe.

\section{DETAILS OF THE EXPERIMENTAL SETUP}

The general outline of the PTD setup is shown in Fig. 5. It consists of two mutually perpendicular frames, one for the pump laser and one for the probe laser, which are rigidly clamped to each other. The crossing point of the pump and probe laser is situated inside the pump laser cavity, to take advantage of the higher local power density. The whole setup is computer controlled and allows unattended measurements for up to one week. Computer control includes tuning of the pump laser to different laser lines and subsequent cavity op- 


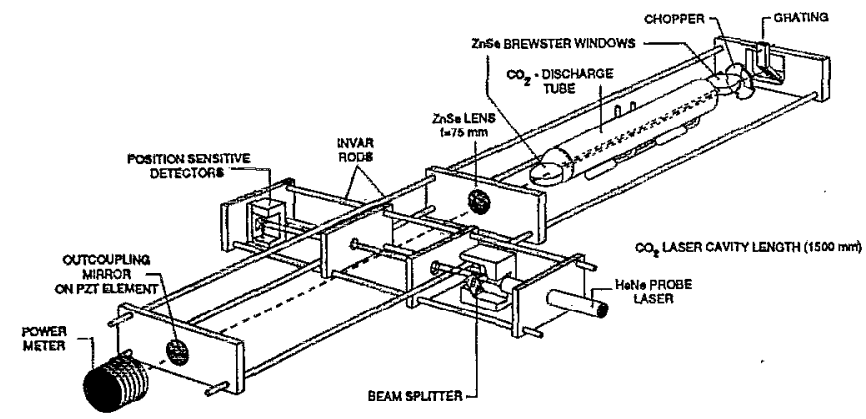

FIG. 5. The photothermal deflection setup, consisting of an intracavity $\mathrm{CO}_{2}$ laser and a double probe beam derived from one HeNe laser. In the crossing region a multipass configuration can be implemented (see Fig. 6 for details).

timization, data acquisition, and on-line data reduction to absolute, local trace gas concentration values.

Technical details are summarized in Table I.

\section{A. Pump laser}

As pump laser, a home-built, line-tunable $\mathrm{CO}_{2}$ waveguide laser is used, based on the proven design of earlier work. ${ }^{39}$ The laser operates on a continuous flow of a gas mixture containing $\mathrm{He}, \mathrm{CO}_{2}$, and $\mathrm{N}_{2}$ (volume ratio of 65:13:22; total pressure 60 mbar) through a quartz waveguide tube ( $400 \mathrm{~mm}$ length, $3 \mathrm{~mm}$ inner diameter) in which a dc discharge is sustained. The waveguide tube is sealed by $\mathrm{ZnSe}$ Brewster windows at both ends; it is cooled by a water jacket, has one central anode, and hollow cathodes at both ends (all electrodes of oxidized nickel). It is positioned close to a plane, gold-coated grating (150 lines $/ \mathrm{mm})$ which serves as tuning element.

The main modification of the laser as compared to the original design ${ }^{39}$ concerns the cavity length, which is extended here to $1200 \mathrm{~mm}$. The cavity is closed by a concave

TABLE I. Technical details of the photothermal deflection setup.

\begin{tabular}{|c|c|}
\hline \multicolumn{2}{|l|}{ Intracavity pump laser setup } \\
\hline Cavity length & $1.5 \mathrm{~m}$ \\
\hline Focal length lens & $75 \mathrm{~mm}$ \\
\hline Beam waist in focus & $0.28 \mathrm{~mm}$ \\
\hline Focal length outcoupling mirror & $135 \mathrm{~mm}$ \\
\hline Reflectivity outcoupling mirror & $98.4 \%$ \\
\hline Intracavity ${ }^{12} \mathrm{CO}_{2}$ laser power & $100 \mathrm{~W}(10 P 14)$ \\
\hline Waveguide tube & $\begin{array}{l}\text { Quartz, water cooled } \\
3 \mathrm{~mm} \text { inner diameter } \\
400 \mathrm{~mm} \text { discharge length }\end{array}$ \\
\hline $\begin{array}{l}\text { Gas mixture } \\
\text { Grating }\end{array}$ & $\begin{array}{l}\text { He: } \mathrm{CO}_{2}: \mathrm{N}_{2}=65: 13: 22 @ 60 \mathrm{mbar} \\
\text { gold coated, } 150 \text { lines } / \mathrm{mm}\end{array}$ \\
\hline Probe laser setup & \\
\hline Probe laser & $\begin{array}{l}\text { HeNe laser }(\lambda=632.8 \mathrm{~nm} \text { ) } \\
\text { (Melles Griot 05-LHP-321) }\end{array}$ \\
\hline Output beam waist & $0.4 \mathrm{~mm}$ \\
\hline Divergence angle & $1 \mathrm{mrad}$ \\
\hline Detector & Quadrant detector (Centronic QD 50-2) \\
\hline Sensitivity & $0.512 \mathrm{~V} / \mathrm{mm}$ \\
\hline Electronic noise & $1.5 \mu \mathrm{V}$ \\
\hline Pointing noise & $3 \mu \mathrm{V}$ \\
\hline Overall noise (single probe beam) & $10 \mu \mathrm{V}$ (at $100 \mathrm{~W}, \mathrm{RC}$-time $1 \mathrm{~s}$ ) \\
\hline Overall noise (double probe beam) & $5 \mu \mathrm{V}$ (at $100 \mathrm{~W}, \mathrm{RC}$-time $1 \mathrm{~s}$ ) \\
\hline
\end{tabular}

ZnSe outcoupling mirror $(f=135 \mathrm{~mm}, R=98.4 \%$ ) mounted on a piezoelectric transducer (PZT) element, which is used for fine tuning the cavity length. Behind the waveguide tube the laser beam is confined by a biconvex ZnSe lens $(f=75$ $\mathrm{mm}$ ), which produces an intracavity focus of $0.28 \mathrm{~mm}$ waist. Between the lens and the outcoupling mirror is ample free space ( $350 \mathrm{~mm}$ length) in which the sample can be placed.

The laser operates on about 90 different lines in the $900-1100 \mathrm{~cm}^{-1}$ region, with typically $100 \mathrm{~W}$ intracavity power on the strongest lines. Accurate laser line frequencies have been published by Bradley et al. ${ }^{40}$ The laser intensity can be amplitude modulated by a mechanical chopper placed between the grating and the waveguide tube.

\section{B. Probe laser}

The current setup employs a red HeNe laser with good pointing stability (Melles Griot 05-LHP-321) as probe laser. Its deflection (in a vertical plane) is detected by a position sensing quadrant detector (Centronic QD50-2). The amplitude modulated pump laser induces a periodical output signal of this quadrant detector, which is fed into a lock-in amplifier (PAR 5204).

The probe laser passes over the intra-cavity pump laser focus either once or in a multipass configuration. The latter involves either two plane mirrors $(R=99.5 \%)$ under an adjustable wedge angle [typically $1^{\circ}-5^{\circ}$; see Fig. 6(a)] or two nearly confocal cylindrical mirrors $[R=99.5 \%$; cylinder axis in the vertical plane; Fig. 6(b)]. Details are summarized in Table II.

To avoid perturbations induced by air turbulence, the probe beam can be split in two beams, the position of each of which is detected by a separate quadrant detector [Hig. 6(c)]. One probe beam passes directly over the pump laser focus (this one is sensitive to a pump laser induced refractive index gradient), the other one passes somewhat further away (and is not affected by the pump laser, but still feels air turbulence in the same way as does the other probe beam). By subtracting the two probe beam signals, the contribution of air turbulence cancels out.

\section{SYSTEM PERFORMANCE}

Typical curves of (single pass) deflection angles as a function of distance between the pump and probe laser and of chopper frequency are illustrated in Fig. 4. All calibration measurements and linearity checks reported below have been done on $\mathrm{C}_{2} \mathrm{H}_{4} /$ air mixtures prepared from a certified 1200 ppbv mixture (supplied by UCAR) at a chopper frequency of $20 \mathrm{~Hz}$, with the pump laser operating on the strongly absorbing $10 P 14$ line $\left(949.479 \mathrm{~cm}^{-1} ; \alpha=13.3 \mathrm{~cm}^{-1}\right.$ for $100 \mathrm{~W}$ laser power (see Table III).

\section{A. Sensitivity and spatial resolution}

At the optimum distance between the pump and probe laser beams $(0.7 \mathrm{~mm})$ the single pass deflection angle amounts to $0.043 \mathrm{~s}$ of arc per $\mathrm{mW}$ laser power absorbed per $\mathrm{cm}$. This value can be improved considerably by using a multipass setup for the probe laser (cf. Figs. 5 and 6). One possible configuration consists of two plane mirrors (flatness 

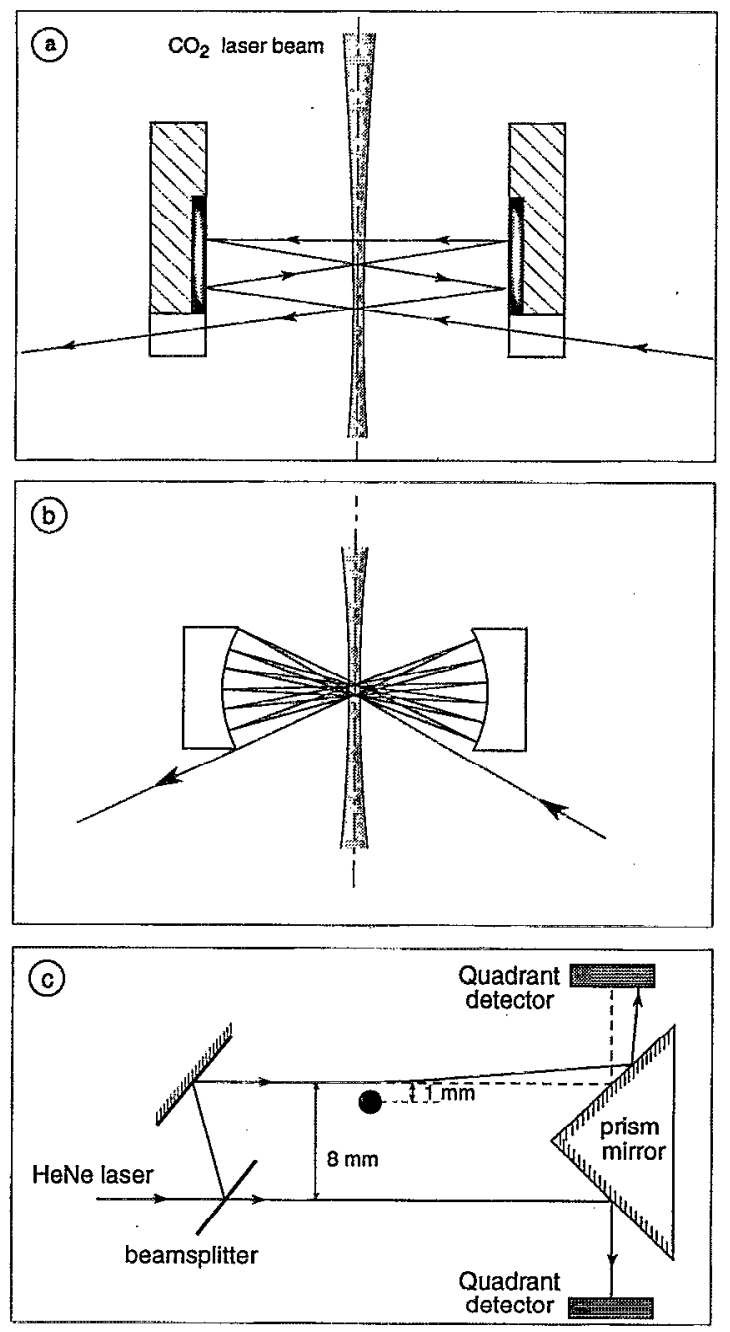

FIG. 6. 'Different probe laser configurations. (a) Multipass arrangement using two slightly tilted plane mirrors. Optimal alignment results in a 20-fold signal gain as compared to the single-pass situation. The spatial resolution along the pump laser beam is $25 \mathrm{~mm}$. (b) Multipass arrangement using two cylindrical mirrors. A factor of 6 gain in sensitivity with respect to the single pass case can be achieved, with a lateral resolution of $2 \mathrm{~mm}$. (c) The double probe beam arrangement. The black dot indicates the pump laser beam. A beam splitter provides a reference beam to correct for air turbulence, as described in the text. This arrangement can be used in parallel with both multipass configurations.

$\lambda / 20 ; R=99.5 \%)$ under a small wedge angle $\theta .{ }^{49}$ The number of passes between these mirrors is a function of both $\theta$ and the initial angle of incidence $\beta$ [Fig. 6(a)]. In practice, the beam divergence and finite beam waist cannot be neglected, and pose a limit both to the maximum number of passes that can be attained and the overall gain in signal-tonoise ratio. Increasing the number of passes leads to a larger deflection angle and to a larger lever arm. The beam displacement $\Delta z$ at the detector site is described by ${ }^{50}$

$$
\Delta z=\frac{1}{2} n^{2} d_{1} \alpha+n d_{2} \alpha,
$$

where $n$ is the number of passes, $\alpha$ the deflection angle per pass, $d_{1}$ the nominal distance between the two mirrors, and $d_{2}$ the distance between the second mirror and the quadrant detector. It has been verified experimentally that the single pass deffection is independent of the lateral position of the
TABLE II. Details of the probe laser configurations used in the experiments. The probed volume has the shape of a cylinder of $0.3 \mathrm{~mm}$ diam and a length given by the lateral resolution (i.e., along the pump laser beam) in the table.

Single pass configuration

Lateral resolution

Sensitivity to $\mathrm{C}_{2} \mathrm{H}_{4}$ (singlc/double beam)

Sensitivity to $\mathrm{NH}_{3}$ (single/double beam)

$4 \mathrm{~mm}$

$20 / 10$ ppbv

10/5 ppbv

Flat mirror setup

Mirror reflectivity

Mirror dimensions

Optimal number of passes

Lateral resolution

Sensitivity to $\mathrm{C}_{2} \mathrm{H}_{4}$ (single/double beam)

Sensitivity to $\mathrm{NH}_{3}$ (single/double beam)

$99.5 \%$

$25 \mathrm{~mm} \times 25 \mathrm{~mm}$

31

$25 \mathrm{~mm}$

$1 / 0.5 \mathrm{ppbv}$

$0.5 / 0.25 \mathrm{ppbv}$

Cylindrical mirror setup

Mirror reflectivity

Mirror dimensions

Mirror focal length

Cyl. lens dimensions

Cyl. lens focal length

Maximum number of passes

Lateral resolution

Sensitivity to $\mathrm{C}_{2} \mathrm{H}_{4}$ (single/double beam)

Sensitivity to $\mathrm{NH}_{3}$ (single/double beam)
$99.5 \%$

$26 \mathrm{~mm} \times 60 \mathrm{~mm}$

$-45 \mathrm{~mm}$

$26 \mathrm{~mm} \times 60 \mathrm{~mm}$

$76.2 \mathrm{~mm}$

15

$6 \mathrm{~mm}$

$3 / 1.5 \mathrm{ppbv}$

$1.5 / 0.75 \mathrm{ppbv}$ probe laser along the pump laser beam over the region covered by the multipass setup. Optimal alignment was found at $n=31$ passes, resulting in a factor of 20 gain in sensitivity as compared to the single pass case.

Using cylindrical mirrors [Fig. 6(b)] and a cylindrical lens to focus the incoming probe beam, a maximum number of 15 passes could be achieved, resulting in a three times lower sensitivity than with the plane mirror system. It should, however, be noted that any such gain in sensitivity is paid for by a decrease in spatial resolution. The latter is determined by the volume heated by the pump laser, which contributes significantly to the probe laser deflection. This volume roughly corresponds to a cylinder with a diameter determined by the pump laser focal width $\left(2 w_{0} \approx 0.6 \mathrm{~mm}\right)$ and a length determined by the effective width of the probe laser, which amounts to $0.8 \mathrm{~mm}$ for the single pass arrangement, $2 \mathrm{~mm}$ using the cylindrical mirror multipass configuration, and $25 \mathrm{~mm}$ with the plane mirror setup [compare Figs. 6(a) and 6(b)]. Data are summarized in Table II.

In all cases, the deflection signal scales linearly with the $\mathrm{C}_{2} \mathrm{H}_{4}$ concentration in the range of $10-1200$ ppbv and with the laser power.

\section{B. Noise sources and the detection limit}

Three main sources of noise can be distinguished in our setup. The noise in the detection electronics typically amounts to $1.5 \mu \mathrm{V} / \sqrt{\mathrm{Hz}}$. The pointing noise of the probe laser, including the contribution of mechanical vibrations of the whole frame, is typically $3 \mu \mathrm{V} / \sqrt{\mathrm{Hz}}$ (plane mirror multipass setup). Although both of these can be reduced by choosing a higher chopper frequency, this would also lead to a reduced deflection amplitude (see Fig. 4). A $20 \mathrm{~Hz}$ chopper frequency was found to be a compromise between these conflicting demands. This value was partly determined by the 
TABLE III. Absorption coefficients (in atm ${ }^{-1} \mathrm{~cm}^{-1}$ ) for $\mathrm{C}_{2} \mathrm{H}_{4}, \mathrm{NH}_{3}, \mathrm{CO}_{2}$, and $\mathrm{H}_{2} \mathrm{O}$ on the ${ }^{\mathrm{I}} \mathrm{CO}_{2}$ laser lines as used in this article. These are air broadened values valid at 1 atm $\left(10^{5} \mathrm{~Pa}\right)$ total pressure; for $\mathrm{C}_{2} \mathrm{H}_{4}$, they have also been corrected for saturation (at $100 \mathrm{~W}$, Ref. 48). For $\mathrm{H}_{2} \mathrm{O}$, average absorption coefficients were calculated.

\begin{tabular}{lccccc}
\hline \hline $\begin{array}{l}{ }^{12} \mathrm{CO}_{2} \\
\text { Laser line }\end{array}$ & $\begin{array}{c}\text { Frequency } \\
\left(\mathrm{cm}^{-1}\right)\end{array}$ & $\mathrm{C}_{2} \mathrm{H}_{4}$ & $\mathrm{NH}_{3}$ & $\begin{array}{c}\mathrm{CO}_{2} \\
\left(\times 10^{3}\right)\end{array}$ & $\begin{array}{c}\mathrm{H}_{2} \mathrm{O} \\
\left(\times 10^{6}\right)\end{array}$ \\
\hline $10 P 14$ & 949.479 & 13.3 & 0.795 & 2.26 & 1.2 \\
$10 P 12$ & 951.192 & 4.3 & 0.663 & 2.15 & 0.95 \\
$10 R 8$ & 967.708 & 0.924 & 20.5 & 1.93 & 0.88 \\
$10 R 10$ & 969.140 & 1.31 & 0.542 & 2.19 & 0.75 \\
$10 R 20$ & 975.930 & 1.09 & 0.0355 & 2.34 & 11.3 \\
Reference & 40 & 41 & 42 & 43 & $44,45,46,47$ \\
\hline \hline
\end{tabular}

presence of building vibrations at lower and higher frequencies, and should be determined empirically at each site where the instrument is applied.

A third contribution to the overall noise comes from air turbulence along the probe laser beam path. The associated density fluctuations directly translate into refractive index gradients and a fluttering HeNe beam. Depending somewhat on the scale of these turbulences, this noise source can be largely suppressed by use of the double probe beam technique discussed above [Fig. 6(c)].

It should be noted that the pump beam does not contribute significantly to the noise because of its indirect coupling to the probe beam. Any (small) fluctuations in power or position of the pump beam are communicated to the probe beam only through thermal diffusion, and, as a consequence, damp out rapidly.

Under laboratory conditions, an overall noise level of 5 $\mu \mathrm{V} / \sqrt{\mathrm{Hz}}$ can be achieved. With a $1 \mathrm{~s}$ integration time and typically $100 \mathrm{~W}$ intra-cavity pump laser power, this corresponds to a detection limit of $6 \times 10^{-9} \mathrm{~cm}^{-1}$ (in terms of the absorption coefficient), using the plane mirror multipass setup with 31 passes and a double probe beam.

\section{Selectivity and response time}

The selectivity of the delector is determined by the extent to which trace gases show a characteristic $\mathrm{CO}_{2}$ laser absorption spectrum (fingerprint spectrum). In favorable cases, the trace gas of interest is the sole contributor to the absorption on one specific $\mathrm{CO}_{2}$ laser line, and deflection measurements on this single line will be unambiguous. Generally, however, the absorption on a given pump laser line will contain contributions both from a (broadband) background and the trace gas of interest. In case of ambient air measurements, the dominant sources of background gas absorption are $\mathrm{CO}_{2}$ and water vapor, with typical concentrations of 310 ppmv and $0.6 \%-3 \%$, respectively. ${ }^{38}$ Both possess a relatively featureless spectrum, ${ }^{43,47}$ with little difference in absorption on neighboring $\mathrm{CO}_{2}$ laser lines. By recording the deflection signals on two laser lines (only one of which should be absorbed by the trace gas), the background absorption can be corrected.

The response time of the setup also depends largely on the amount of background absorption present. Preferably, a pump laser line which is significantly absorbed only by the trace gas of interest should be selected. In this case, measure- ments on a single pump laser line will be sufficient, and the response time will be determined by the integration time required to get a reasonable signal-to-noise ratio. A fundamental limit is posed, however, by the thermal diffusion time connecting pump and probe laser ( $15 \mathrm{~ms} @ \Delta z=0.7 \mathrm{~mm}$ ).

When background absorption plays a major role, the deflection signals on two different pump laser lines should be compared. These should be selected such that the background absorption be similar on both lines, but the trace gas absorption contribute to one line only. In our current setup, the overall response time, in this case, is determined by the $15 \mathrm{~s}$ required to switch between two pump laser lines and re-optimalization of the cavity length.

These aspects will be exemplified by two applications discussed below.

\section{TWO APPLICATIONS}

\section{A. Localization of ethylene emission sites on ripening cherry tomatoes}

The important role of ethylene, the only gaseous plant hormone, in plant physiology is well documented in the literature; an extensive survey is provided by Abeles et al. ${ }^{26}$ Ethylene possesses a distinctive fingerprint spectrum in the ${ }^{12} \mathrm{CO}_{2}$ laser range; air broadened absorption cocfficients at atmospheric pressure have been published by Brewer. ${ }^{41}$ For selective ethylene detection, the strong, isolated absorption on the $10 P 14$ line can be used. The neighboring, less absorbing $10 P 12$ line lends itself for background correction. $\mathrm{Ab}$ sorption coefficients are summarized in Table III.

We have applied the photothermal detection technique to locate the ethylene emission sites of cherry tomatoes ( $\mathrm{LyCO}$ persicon esculentum 'Cherry'). Intact cherry tomatoes were in the mature orange stage, with a weight of approximately $14 \mathrm{~g}$ and about $35 \mathrm{~mm}$ diam. They were detached from the plant and, in most cases, the coronet (including pedicel and sepals) was removed. Tomatoes were placed under the crossing of the two laser beams $1.5 \mathrm{~mm}$ below the pump laser. This was sufficiently far away to avoid heating the skin. Measurements, carried out at $21^{\circ} \mathrm{C}$ under normal atmospheric conditions, were performed at the stem-scar site (coronet site), the bottom site (opposite to the stem-scar site), and an equatorial site. The data are presented in Fig. 7(a). Evidently, most of the internally produced ethylene is released at the coronet site $(90 \%)$. The amounts of ethylene 

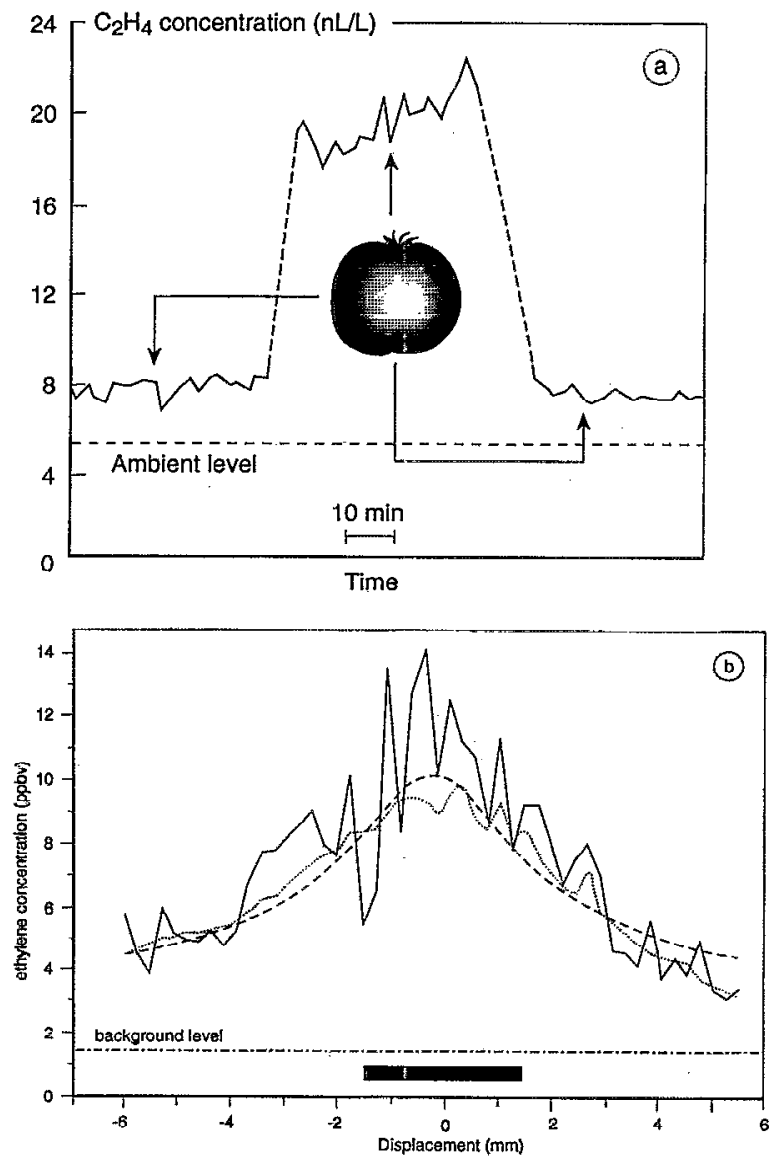

FIG. 7. Ethylene emission by a cherry tomato. (a) Ethylene concentrations as measured at $1.5 \mathrm{~mm}$ from the surface of a green/orange tomato above an equatorial site, the stem scar and the bottom site. (b) Instantaneous ethylene concentrations in a horizontal plane above the stem-scar region (solid line) and the average concentrations (average of 22 scans; dotted line). A fit of the average concentration to $\mathrm{Eq}$. (18) is also incorporated (dashed line). The minimum distance between tomato surface and $\mathrm{CO}_{2}$ laser is $1.5 \mathrm{~mm}$ (at $z=0$ ). The diameter of the stem-scar area is indicated by a bold bar.

emitted at the bottom site and at the equator both are very low, just above ambient $(\approx 4 \mathrm{ppbv})$.

Our estimate of $90 \%$ of internally produced ethylene being released through the stem-scar area is in general agreement with values obtained by indirect methods. By comparing the total ethylene emission of untreated tomatoes with that of tomatoes of which the stem scar was blocked with lanolin paste, Burg and $\mathrm{Burg}^{28}$ estimated that $60 \%$ of the internally produced ethylene was emitted through the stem scar. However, blocking the stem scar is accompanied by oxygen deprivation of the fruit interior and, therefore; necessarily results in a perturbed metabolism. ${ }^{51}$

Cameron and Yang ${ }^{29}$ determined the resistance to ethane effusion of the stem scar, relative to that of the skin, by preloading a normal size tomato with high concentrations of ethane and determining the subsequent ethane eflux out of the stem scar and out of the rest of the fruit. However, in their skin resistance model the production of ethylene in different parts of the fruit has not been considered, which might be important since at different stages of maturity different parts of a tomato produce very different amounts of ethylene. ${ }^{52}$
Using the cylindrical mirror setup, the ethylene concentration profile in a horizontal plane above the tomato stem scar was determined [Fig. 7(b)]. The resulting profile corresponds to what would be expected from radial diffusion out of a point source. This is described by ${ }^{33}$

$$
\rho(r, t)=\frac{\Phi}{4 \pi D r},
$$

with $\rho(r, t)$ the local ethylene density, $\Phi$ the point source production rate $\left(\mathrm{m}^{3} / \mathrm{s}\right), D$ the diffusion coefficient $\left(1.8 \times 10^{-5}\right.$ $\mathrm{m}^{2} \mathrm{~s}^{-1}$ for ethylene in air $^{53}$ ), and $r$ the distance from the source. From this equation, the measured concentrations can be related to a (point source) production rate.

Note that the fluctuations in ethylene concentration observed in Fig. 7(b) are not due to noise, but are the result of real concentration changes during the measurement.

\section{B. On-line monitoring of ammonia concentrations}

Quantitative measurements of ammonia concentrations are hampered by memory effects hecause $\mathrm{NH}_{3}$, with its large permanent dipole moment, easily sticks to any surface it encounters. Sensitive detectors, based on active sampling and with response times of several minutes, have been constructed using photoacoustical ${ }^{48}$ and wet chemical ${ }^{54}$ methods.

Photothermal deflection, although not (yet) as sensitive as the above mentioned techniques, has the evident advantage of contact-free measurement, thus avoiding all memory effects. Like $\mathrm{C}_{2} \mathrm{H}_{4}$, ammonia also possesses a distinct fingerprint spectrum in the ${ }^{12} \mathrm{CO}_{2}$ laser range. Absorption strengths are given by Brewer ${ }^{42}$ and are summarized in Table III. We use the $10 R 8$ for $\mathrm{NH}_{3}$ detection and the adjacent $10 R 10$ for background correction.

In order to examine the response time of the PTD instrument, short pulses of ammonia-enriched air were flushed through the detection area. The air was introduced through narrow teflon tubing, the opening of which was positioned $20 \mathrm{~mm}$ below the pump laser beam. With the $\mathrm{CO}_{2}$ laser fixed on the $10 R 8$, the rise time of the ammonia signal (see Fig. 8) is essentially determined by the limited flow rate at which the ammonia-enriched air could be supplied. As shown by subsequent measurements on the $10 R 10$, the background signal level is virtually unperturbed by the enriched air pulses. With the present setup, fastest response times for single line measurements amount to $100 \mathrm{~ms}$, determined by the integration time of the lock-in detection electronics. This renders the system fast enough to be incorporated in eddy correlation measurements for monitoring dry deposition rates. ${ }^{32}$

Apart from its role in soil acidification, already alluded to in the introduction, ammonia is also of importance because it is an intermediate in nitrogen fixation. ${ }^{55}$ Cyanobacteria, or blue-green algae, belong to the few organisms capable of fixing atmospheric nitrogen. ${ }^{56,57}$ The first step in nitrogen fixation proceeds by the reduction of $\mathrm{N}_{2}$ to $\mathrm{NH}_{3}$, catalyzed by the enzyme nitrogenase. Normally, the ammonia remains dissolved in the cell interior, where it is rapidly metabolized into other, less toxic compounds. The main pathway of this second step is the glutamine synthetase ca- 


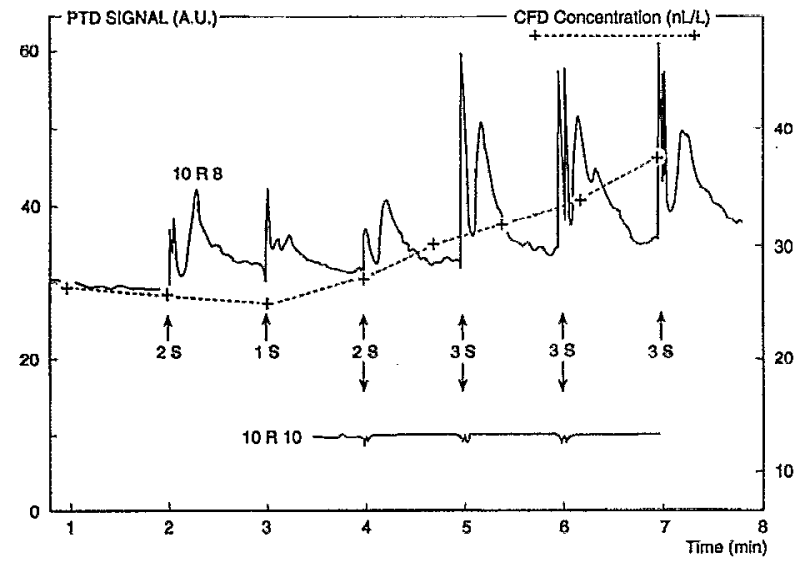

FIG. 8. Ammonia-enriched air pulses. A constant flow of either normal air or ammonia-enriched air is directed towards the detection region. The duration of each ammonia-enriched air pulse is indicated; the effective switching time is limited by the teflon tubing used to guide the flow. Measurements are taken on the $10 R 8$; the background level $(10 R 10)$ is seen to be virtually unperturbed by switching between the two types of flow. Also included is the response of a continuous flow denuder (CFD; dashed line). The fine structure of the peaks measured by the PTD setup is due to details of the flow system.)

talysed conversion of glutamic acid and ammonia into glutamine (GOGAT pathway ${ }^{58}$ ). This step can be blocked by the glutamate analog MSX ( $L$-methionine- $D, L$ sulphoximine) without concomitant effect on nitrogenase activity. $^{59-61}$

Generally, nitrogenase activity is demonstrated by expluiling the fact that this enzyme also catalyzes the reduction of acetylene to ethylene. ${ }^{62-64}$ By applying a well-defined amount of acetylene to a cyanobacteria culture and determining the ethylene concentration after an incubation time, nitrogenase activity can be determined. This, in turn, can be used to estimate the nitrogen fixation rate via a conversion factor which may depend on external conditions. ${ }^{64,65}$

Photothermal deflection allows us to directly measure the absolute ammonia concentration in the air above a cyanobacteria culture. We performed some experiments on a Gloeothece strain (PCC 6909, Pasteur Culture Collection, Paris), grown diazotrophically under artificial light in BG $11^{\circ} .{ }^{66}$ Cell suspensions were transferred on a filter paper support to small Petri dishes, which were placed $1.5 \mathrm{~mm}$ below the $\mathrm{CO}_{2}$ laser beam in the detection region.

Ammonia levels in the air above such a culture were equal to ambient, indicating that $\mathrm{NH}_{3}$, if formed at all, remains in solution. Proof that ammonia is indeed produced is provided by adding an overdose of $\mathrm{NaOH}$ to the medium, thus driving out all dissolved $\mathrm{NH}_{3}$; this resulted in a large, transient rise of the ammonia concentration above the culture, as shown in Fig. 9(a). The initial, steep rise is probably due to ammonia escaping from the liquid culture medium. Afterwards, the level is maintained by the slower expulsion of the ammonia dissolved in the cell interior.

Adding MSX $(50 \mu \mathrm{M})$ to a culture also results in a transient rise of the ammonia concentration above the culture, as can be seen from Fig. 9(b). The time lag observed between the application of MSX and the rise in ambient air ammonia content can probably be attributed to the buffer capacity of
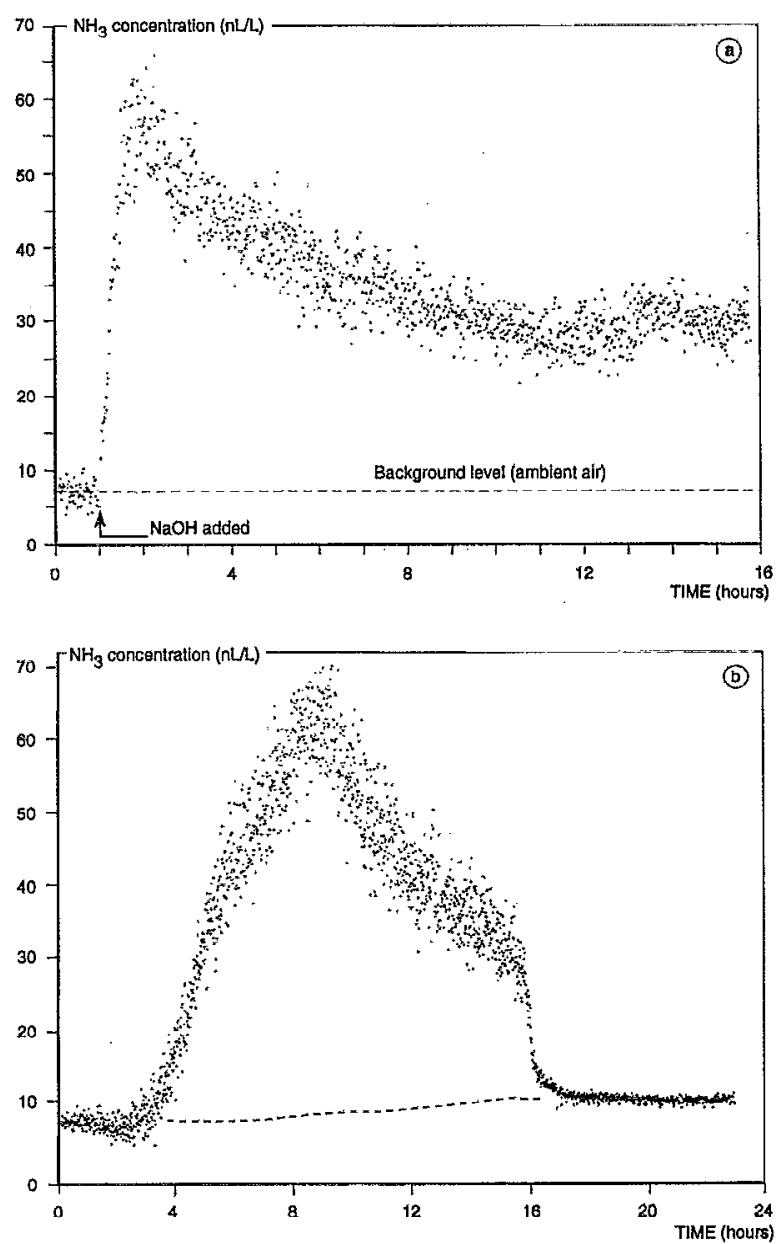

FIG. 9. Ammonia production by nitrogen fixing cyanobacteria (Glvevthece PCC 6909) cultured on nitrogen-free medium. (a) Untreated cyanobacteria cultures do not produce measurable concentrations of free ammonia $(t<1$ h). When dissolved ammonia is driven out (by adding an overdose of $\mathrm{NaOH}$ to the culture, at $t=1 \mathrm{~h}$ ), a large peak results which declines only slowly. (b) When MSX is added to a culture (at $t=0$ ) to block intracellular ammonia conversion, the $\mathrm{NH}_{3}$ concentration above the culture is seen to rise after a time lag of about $3 \mathrm{~h}$. The subsequent decline is probably due to drying out of the culture.

the liquid medium and/or the cyanobacteria themselves. The gradual decline of the $\mathrm{NH}_{3}$ production after $t=8 \mathrm{~h}$ is due most probably to the slow desiccation of the culture. Rewetting resulted in resumed $\mathrm{NH}_{3}$ production. ${ }^{67}$ Note that the "noise" in the measurements (datapoints taken every minute) is larger at the beginning of the experiment $(t=0-3$ $\mathrm{h}$; living, active culture) than at the end ( $t>16 \mathrm{~h}$; living, dormant culture). Evidently, although the average ammonia release from an untreated Gloeothece sample is too small to be detected by our method, its fluctuations lead to clearly observable spikes on the ambient ammonia concentration. More detailed experiments on the nitrogen fixation rates of cyanobacteria under different environmental conditions are in progress.

\section{ACKNOWLEDGMENTS}

The authors gratefully acknowledge L. Voesenek, C. Blom, E. Woltering, H. van der Valk, and I. Stal for discus- 
sions concerning the biological aspects of this work and $\mathrm{J}$. Holtkamp, F. van Rijn, and C. Timmer for expert technical assistance.

${ }^{1}$ O. E. Schupp III, in Technique of Organic Chemistry, edited by E. S. Perry and A. Weissberger (Interscience, Wiley, New York, 1968), Vol. 13.

${ }^{2}$ A. B. Littlewood, Gas Chromatography; Principles, Techniques and Applications, 2nd ed. (Academic, New York, 1970).

${ }^{3}$ A. C. Tam, Rev. Mod. Phys. 58, 381 (1986).

${ }^{4}$ S. Bernegger and M. W. Sigrist, Infrared Phys. 30, 375 (1990).

${ }^{5}$ P. L. Meyer and M. W. Sigrist, Rev. Sci. Instrum. 61, 1779 (1990).

${ }^{6}$ F. G. C. Bijnen, H. S. M. de Vries, F. J. M. Harren, and J. Reuss, J. Phys. (Paris) C 4, 435 (1994).

${ }^{7}$ Photoacoustic and Photothermal Phenomena III, Springer Series in Optìcal Sciences, Vol. 69, edited by D. Bicanic (Springer, Berlin, 1992).

${ }^{8}$ N. M. Amer, SPIE 794, 11 (1987).

${ }^{9}$ R. C. Frye, J. J. Kumler, and C. C. Wong, Appl. Phys. Lett. 50, 101 (1987).

${ }^{10}$ E. Sauvain, A. Mettler, N. Wyrsch, and A. Shah, Solid State Commun. 85, 219 (1993).

${ }^{11}$ J. D. Spear, R. J. Silva, G. L. Klunder, and R. E. Russo, Appl. Spectrosc. 47, 1580 (1993).

${ }^{12}$ W. Faubel, T. Schultz, B. S. Seidel, E. Stcinle, and H. J. Achc, J. Phys. (Paris) C 4, 531 (1994).

${ }^{13}$ A. C. Boccara, D. Fournier, W. Jackson, and N. M. Amer, Opt. Lett. 5, 377 (1980).

${ }^{14} \mathrm{~K}$. Rajasree, V. Vidyalal, P. Radhakrishnan, V. P. N. Nampoori, and C. P. G. Vallabhan, J. Appl. Phys. 73, 2004 (1993).

${ }^{15}$ A. Salazar, A. Sanchezlavega, and J. Fernandez, J. Appl. Phys. 74, 1539 (1993).

${ }^{16}$ M. A. Shannon, B. Rubinsky, and R. E. Russo, J. Appl. Phys. 75, 1473 (1994).

${ }^{17}$ K. Plamann, K. Giese, B. Sennhenn, N. Harendt, and K. Kölmel, in Ref. 7, p. 92.

${ }^{18}$ S. Matsuzawa, H. Kimura, C. Y. Tu, T. Kitamori, and T. Sawada, J. Immunological Methods 161, 59 (1993).

${ }^{19}$ J. A. Sell and R. J. Cattolica, Appl. Opt. 25, 1420 (1986).

${ }^{20}$ T. M. Brown, R. W. Pitz, C. P. Hess, and C. P. Wood, Appl. Phys. B 59, 351 (1994).

${ }^{21}$ D. Fournier, A. C. Boccara, N. M. Amer, and R. Gerlach, Appl. Phys. Lett. 37, 519 (1980).

${ }^{22}$ K. H. Fung and H. B. Lin, Appl. Opt. 25, 749 (1986).

${ }^{23}$ J. C. Murphy and L. C. Aamodt, J. Appl. Phys. 51, 4580 (1980).

${ }^{24}$ W. B. Jackson, N. M. Amer, A. C. Boccara, and D. Fournier, Appl. Opt. 20, 1333 (1981).

${ }^{25} \mathrm{~F}$. Charbonnier and D. Fournier, Rev. Sci. Instrum. 57, 1126 (1986).

${ }^{26}$ F. B. Abeles, P. W. Morgan, and M. E. Saltveit Jr., Ethylene in Plant Biology, 2nd ed. (Academic, London, 1992).

${ }^{27}$ S. P. Burg and E. A. Burg, Bot. Gaz. 126, 200 (1965).

${ }^{28}$ S. P. Burg and E. A. Burg, Physiol. Plants 18, 870 (1965).

${ }^{29}$ A. C. Cameron and S. F. Yang, Plant Physiol. 70, 21 (1982).

${ }^{30}$ M. E. Saltveit, Jr., Physiol. Plants 89, 204 (1993).

${ }^{31}$ L. J. M. van der Eerden, Agric. Environm. 7, 223 (1982).

${ }^{32} \mathrm{~J}$. Slanina (private communications).

${ }^{33}$ J. Crank, The Mathematics of Diffusion, 2nd ed. (Oxford University Press, Oxford, 1975).
${ }^{34}$ The absorption coefficient $\alpha$ is related to the absorption cross-section $\sigma$ by $\alpha=\sigma \rho_{a} / p_{a}$.

${ }^{35}$ W. Demtröder, Laser Spectroscopy, 2nd ed., Springer Series in Chemical Physics, Vol. 5 (Springer, Berlin, 1982).

${ }^{36}$ The validity of this assumption has been verified experimentally by varying the lateral position of the probe laser with respect to the pump laser focus.

${ }^{37}$ M. Abramowitz and I. A. Stegun, Handbook of Mathematical Functions (Dover, New York, 1970).

${ }^{38}$ Handbook of Chemistry and Physics, 73rd ed., edited by D. R. Lide (CRC, Boca Raton, 1992).

${ }^{39}$ F. J. M. Harren, J. Reuss, E. J. Woltering, and D. D. Bicanic, Appl. Spectrosc. 44, 1360 (1990).

${ }^{40}$ L. C. Bradley, K. L. Soohoo, and C. Freed, IEEE J. Quantum Electron. 22, 234 (1986)

${ }^{41}$ R. J. Brewer, C. W. Bruce, and J. L. Mater, Appl. Opt. 21, 4092 (1982).

${ }^{42}$ R. J. Brewer and C. W. Bruce, Appl. Opt. 17, 3746 (1978).

${ }^{43}$ Hitran database (1991).

${ }^{44}$ M. S. Shumate, R. T. Menzies, J. S. Margolis, and L. G. Rosengren, Appl. Opt. 15, 2480 (1976).

${ }^{45}$ G. L. Loper, W. A. O'Neal, and J. A. Gelbwachs, Appl. Opt. 22, 3701 (1983).

${ }^{46}$ J. S. Ryan, M. H. Hubert, and R. A. Crane, Appl. Opt. 23, 781 (1983).

${ }^{41}$ W. B. Grant, Appl. Opt. 29, 451 (1990).

${ }^{48}$ F. J. M. Harren, F. G. C. Bijnen, J. Reuss, L. A. C. J. Voesenek, and C. W. P. M. Blom, Appl. Phys. B 50, 137 (1990).

${ }^{49}$ P. G. Lethbridge and A. J. Stace, Rev. Sci. Instrum. 58, 2238 (1987).

${ }^{50}$ H. S. M. de Vries, F. J. M. Harren, and J. Reuss, in Ref. 7, p. 12.

${ }^{51}$ H. S. M. de Vries, E. J. M. Harren, L. A. C. J. Voesenek, C. W. P. M. Blom, E. J. Woltering, H. C. P. M. van der Valk, and J. Reuss, Plant Physíol. (to be published)

${ }^{52}$ J. K. Brecht, Hortic. Sci. 22, 476 (1987).

${ }^{53}$ Landolt-Börnstein, Zahlenwerte und Funktionen, Band $\mathrm{I}$, Teil 5a (Springer, Berlin, 1969).

${ }^{54}$ G. P. Wyers, R. P. Otjes, and J. Slanina, Atmos. Environ. A 27, 2085 (1993).

${ }^{55} \mathrm{~J}$. R. Postgate, The Fundamentals of Nitrogen Fixation (Cambridge University, Cambridge, 1982).

${ }^{56}$ L. J. Stal, Methods Enzymol. 167, 474 (1988).

${ }^{57}$ N. G. Carr and B. A. Whitton, The Biology of Cyanobacteria, 2nd ed., Botanical Monographs, Vol. 19 (Blackwell, Oxford, 1982).

${ }^{58}$ D. Kleiner, S. Phillips, and E. Fitzke, in Biology of Inorganic Nitrogen and Sulphur, edited by H. Bothe and A. Trebst (Springer, Berlin, 1981), p. 131.

${ }^{59}$ W. D. P. Stewart and P. Rowell, Biochem. Biophys. Res. Commun. 65, 846 (1975).

${ }^{60}$ A. Meister, Science 220, 472 (1983).

${ }^{51}$ D. Rhodes, L. Deal, P. Haworth, G. C. Jamieson, C. C. Reuter, and M. C. Erickson, Plant Physiol. 82, 1057 (1986).

${ }^{62}$ R. W. F. Hardy, R. D. Holsten, E. K. Jackson, and R. C. Burns, Plant Physiol. 43, 1185 (1968).

${ }^{63}$ L. J. Stal and W. E. Krumbein, Arch. Microbiol. 143, 67 (1985)

${ }^{64}$ J. K. Vessey, Plant and Soil 158, 151 (1994).

${ }^{65}$ B. B. Jensen and R. P. Cox, Appl. Environm. Microbiol. 45, 1331 (1983).

${ }^{66}$ R. Rippka, J. Deruelles, J. B. Waterbury, M. Herdman, and R. Y. Stanier, J. Gen. Microbiol. 111, 1 (1979).

${ }^{67}$ H. S. M. de Vries, Ph.D. thesis, University of Nijmegen, 1994. 